\title{
Study Scale of the Linear Transformation On Nursing Technology
}

\author{
Jui-Chiao Tseng ${ }^{1, a}$, Ting-Hung Huang ${ }^{1, b}$ and Yuan-Horng Lin ${ }^{2, c}$ \\ ${ }^{1}$ Min-Hwei Junior College of Health Care Management, Taiwan \\ ${ }^{2}$ National Tai-Chung University of Education, Taiwan \\ a jctseng@mail.mhchcm.edu.tw, ${ }^{b}$ thhuang@mail.mhchcm.edu.tw, ${ }^{\circ}$ lyh@mail.ntcu.edu.tw
}

\begin{abstract}
Keywords: Nursing Technology, Yes/No Angoff, Scale of the Linear Transformation.
\end{abstract}
\begin{abstract}
Unified set the passing score of 60 points for Registered Nursing subjects of Professional and Technical Examination. This situation has caused the subjects of fairness concerns. Therefore standardized set by the method to maintain the stability of the threshold of every subject is very necessary. Apply non-equivalent groups with anchor test's design, to proceed the linking of horizontal equating with common items, Follows the equation of Mean/Mean ruler to compute the scale of the linear transformation from the two type assessment test papers. Discuss the parameters of items, parameters of linear transformation about two type assessment test papers. Estimate the above relation parameters and the results of horizontal equating. Finally, Use the method of Yes/No Angoff to construct the standardized set of threshold for registered nurse of entrance exam. The purpose of this study is to investigate the reference data about the threshold criteria and give a reliable threshold of Professional and Technical Examination for Registered Nurse.
\end{abstract}

\section{Introduction}

ICN (International Council of Nursing) in 2001 proposed general-care nurses international standard architecture, nurses should have the license is a country with a government guarantee of its nationals to accept an essential safeguard health care services when (Smith, 2005). "License" is a qualification certificate to prove that have engaged in some kind of special-ized vocational ability, but also gives a personal li-cense to perform his professional (Scotto, 2003). Ability to practice nursing care license certificate, there are nursing license system in order to guarantee the safety of people for medical treatment.

Taiwan Care Nursing Education Evaluation Committee evaluation Care Planning Group graduates should have the basic core of professional accomplishment, critical thinking and reasoning, general clinical nursing skills, basic biomedical science, communication and teamwork capability, caring, ethics, accountability and life-long learning, to the hospital as a school of Nursing planning curriculum, teacher education and the basis for teaching, but also to become foster in our basic goal of professional competence and attitude.

Technical college entrance exam nurse, for example, is required to be up to 60 points pamphlets side to pass, can be applied to detect the extent of the ruler of candidates. Scale scores There are many different forms of computing, calculated from the standard paper "number of questions", all the subjects of "The average number of correct answers", and "standard deviation" calculated based on test results. Mode of operation can be used to convert raw scores can also be taken directly measuring stick when scaling scores. This score is calculated, are less likely to be affected by the emergence of easy questions extreme results. Through the way to scale scores, the degree of conversion of subjects, through fine-tuning scale scores, relatively better performance will likely pass after trimming to avoid regret Pearl's.

Do not number your paper: All manuscripts must be in English, also the table and figure, otherwise we cannot publish your paper. Please keep a second copy of your manuscript in your office. When receiving the paper, we assume that the corresponding authors grant us the copyright to use the paper for the book or journal in question. 


\section{Literature References}

Use classical test theory and item response theory to estimate nursing ability, difficulty of item, identification of the parameters, the parameters of nursing ability, and so on. According to the subjects complete the two type assessment test papers, Researchers collected the response credible data completely, Apply non-equivalent groups with anchor test design, to proceed the linking of horizontal equating with common items, Follows the equation of Mean/Mean ruler to compute the scale of the linear transformation from the two type assessment test papers. Establishing ruler across different grades such as longitudinal or transverse ruler, the amount of assessment tools were necessary; and in the context of this application, vertical, etc. of information to collect data according to different design, divided into longitudinal data and cross-sectional data.

The quiz is a data conversion unit to another unit test data, converted to the equivalent of two type fraction, which is the transformation of the conversion process (Angoff ,1971). Test scores and other technology should not be subject to a certain extent the ability of the subjects have different effects in different versions of the test sometimes accepted. The purpose of the test is to allow equalization between each test scores can be objectively and effectively Comparison to determine a time tested competency standards qualified participants maintain consistency. At present, such as technology has been widely used in various domestic and professional licenses, and achievement test in the language (Lord,1980). Surveying the test when the data were collected equalization, both test scores and other parameters of the questions or the links, can be of different quizzes designed to achieve the purpose of equalization (Vale, 1986). Through the anchor test of design, construction survey carried out for different groups, you can also compare various types of test is homogeneous, and the differences between groups(Klein \& Jarjoura, 1985) Follows the equation of Mean/Mean ruler to compute the scale of the lin-ear transformation from the two type assessment test papers.

The main steps of mean/mean ruler conversion method as following:

$$
\begin{gathered}
\theta_{B}=\alpha \theta_{A}+\beta \\
a_{B}=\frac{a_{A}}{\alpha} \\
b_{B}=\alpha b_{A}+\beta \\
\alpha=\frac{\theta_{B}-\theta_{B}^{*}}{\theta_{A}-\theta_{A}^{*}}=\frac{b_{B}-b_{B}^{*}}{b_{A}-b_{A}^{*}}=\frac{a_{B}}{a_{A}} \\
\beta=b_{B}-\alpha b_{A}=\theta_{B}-\alpha \theta_{A}
\end{gathered}
$$

Average number of anchor test ease of application parameters to calculate link coefficients $A$ and B. A and B tests using test parameters identification of the parameters a and $b$ of the average ease of calculation A quizzes and quiz questions parameter B ruler converted slope and intercept $\alpha$ and $\beta$. Constants $\alpha$ and $\beta$ can be equalized between two different questions formula estimated ability value, determined by a simple algebraic (Loyd \& Hoover,1980).

\section{Experiment of Real Data}

Select nursing graduates as surveying sample, which have 713 nursing graduates of universities and colleges. We have the 20 questions to test the ability to anchor each title Branch of value and differentiation degree $\alpha$ and $\beta$, as shown in Table 1 .

Table $1 \alpha$ and $\beta$ about five subjects of registered nursing exam.

\begin{tabular}{cccccc}
\hline & Basic Nursing & Basic Medical & Surgical Nursing & Perinatal Nursing & Community spirit \\
\hline$\alpha$ & 0.85 & 1.44 & 1.24 & 1.22 & 0.99 \\
$\beta$ & -0.41 & -0.83 & 0.22 & 0.14 & -0.29 \\
\hline
\end{tabular}

Professional and Technical Examination for Registered Nurse over the years average pass rate exist large differences, The possible reasons may be affected by difficulty of item, This led to the 
adoption threshold varies annually, Unified set the passing score of 60 points for Registered Nursing subjects of Professional and Technical Examination.

This situation has caused the subjects of fairness concerns. Therefore standardized set by the method to maintain the stability of the threshold of every subject is very necessary.

First using the chi-square test of the cross table, respectively, the second type test for whether passing by first base after the conversion made as the first variables, the subjects participated in the test of whether pass the professional and technical examination for registered nurse has made a second variables, As shown in Table 2.

Table 2 Cross Table about the Random Sample

\begin{tabular}{lcc}
\hline Scale of the Linear Transformation base on the first type test & $<60$ & $\geq 60$ \\
\hline Not obtained Registered Nurse & 71 & 0 \\
Obtained Registered Nurse & 5 & 265 \\
\hline
\end{tabular}

265 samples of scores over 60 points comes from the second type test which scale of the linear trans-formation base on the first type test, $100 \%$ obtained entrance examination nurse qualifications, There are 5 samples obtain entrance qualifications come from 76 with second type test is for those who have not passed the basis of the conversion, Therefore con-firmed by the second type test for subjects converted basis, $100 \%$ predict the registered nurse entrance examination will be able to get qualified nurse.

Using distinguish function to confirm the difference of them, which is similar to the regression equation to classify of them. The first variables made by scale of the linear transformation base on the first type test, the subjects whether pass the examination for registered nurse made second variables, we get classification results. Available $98.5 \%$ of correct classification, also on the cross-validation group observations have $98.5 \%$ of correct classification, the difference between the function $=11.035$, its canonical correlation $=0.958$, chi-square difference between the value of the function $=842.132$, and reached statistical significant $\left(\mathrm{p}^{* * *}<0.001\right)$.

Use regression analysis, the second type test for whether passing by first base after the converse made as independent variables to predict the criterion variables for whether pass the professional and technical examination for registered nurse. As shown in Table 3, We get the Regression equation is the following equation : $y=0.934 \cdot x+0.066$.

We got the Regression analysis of explanatory power equal to $95.8 \%$ and reached statistical significant $\left(\mathrm{p}^{* * *<0.001)}\right.$.

Table 3 Mode Coefficient of Regression Analysis

\begin{tabular}{lcclcc}
\hline & Estimate value & Standard error & Beta value & $\mathrm{t}$ & Sign. \\
\hline $\mathrm{C}$ & .066 & .013 & 1.24 & & .000 \\
$\mathrm{X}$ & .934 & .015 & 0.22 & .958 & .000 \\
\hline
\end{tabular}

As shown in Table 4, the analysis results, the standard setting decision scores three members of the "basic medicine" has reached the significant difference $(F=9.068, p<.01)$. Have different scores in each round of the decision to set standard statistical members.

Table 4 ANOVA For Standard set of scores in three rounds of decision

\begin{tabular}{ccccc}
\hline Sources of variation & SS & DF & MS & F值 \\
\hline Between groups & 68.96 & 2 & 34.479 & $9.07^{* *}$ \\
Within the group & 45.63 & 12 & 3.802 & \\
Total & 114.58 & 14 & & \\
\hline
\end{tabular}

Therefore, in order to further investigate the case of differences between the scores of each round decision why, researchers used LSD method for post hoc comparisons. 
Table 5 Each round scores compare the summary table

\begin{tabular}{lccc}
\hline Decision Score & First round & Second round & Third round \\
\hline First round $x=66.00$ & --- & --- & --- \\
Second round $x=63.25$ & $-2.75^{*}$ & --- & --- \\
Third round $x=60.75$ & $-5.25^{*}$ & $-2.50^{\text {n..s. }}$ & --- \\
\hline
\end{tabular}

As shown in Table 5, Standard setting members in decision points "third round" and the "first round" decision points up significant differences $(\mathrm{p}<.05)$; and standard-setting member at the "second round" of decision points and at the "first round "decision points up significant differences $(\mathrm{p}<.05)$, terms of the first round as a benchmark, the standard set in the second round with the members of the third round compared to the first round were reached statistically significant differences, and standard setting members less than significant difference ( $p>.05)$ statistical decision points in the second round and the third round of the members in the decision to display the standard set have converged in the third round. Hence the method of Yes/No Angoff to construct the standardized set of threshold is valuable and perfect.

\section{Summary}

Researchers collected the response credible data completely, Apply non-equivalent groups with anchor test design, to proceed the linking of horizontal equating with common items, Follows the equation of Mean/Mean ruler to compute the scale of the linear transformation from the two type assessment test papers. With the above four different algorithms have received a high accuracy. Its average accuracy rate of over $98 \%$ and reached statistical significant $(\mathrm{p} * * *<0.001)$.

Therefore, According to the findings, You can use edit of the two type assessment test papers to predict the correct preparation of registered nurse about graduate of college or university students.

The ruler of the linear transformation value or the ability of the subjects to compare test scores, the ability to value IRT- $\theta$ and Yes/No Angoff method designed to obtain technical college entrance exam nursing license standard setting, as set out decision is consistent scores. The decision set out to verify the validity of the scores obtained, this result is reli-able indeed. This score is calculated, are less likely to be affected by the emergence of easy questions extreme results. Through the way to scale scores, the degree of con-version of subjects, through fine-tuning scale scores, relatively better performance will likely pass after trimming to avoid regret Pearl's. Use the method of Yes/No Angoff to construct the standardized set of threshold for registered nurse of entrance exam is very exactly.

\section{References}

[1]Livingston, S. A.(2004). Equating test scores. Retrieved March 16, 2014, from http://www.ets.org/ Media/Research/pdf/livingston.pdf.

[2] Kolen, M. J. \& Brennan, R. L. (2004). Test equating, scaling, and linking: methods and practices (2nd ed.). New York: Springer-Verlag.

[3] Smith, F. (2005). National service frameworks: from policy to practice. Paediatric Nursing, 16, 32-34.

[4] Scotto, F. J. (2003). A new view of caring. Journal of Nursing Education 42(7), 289-291.

[5] Hamblenton, R. K. \& Swaminathan, H. (1985). Item response theory: principles and applications. Boston: Kluwer Nijhoff Publishing.

[6] Kolen, M. J. \& Brennan, R. L. (2004). Test equating, scaling, and linking: methods and practices (2nd ed.). New York: Springer-Verlag.

[7] Angoff, W. H. (1971). Scales, norms, and equivalent scores. In R.L. Thorridike (Ed.), Educational measurement (2nd ed., 508-600). Washington, DC: American Council on Education. (Reprinted as W. A. Angoff, Scales, norms, and equivalent scores. Princeton, NJ: Educational Testing Service, 1984.)

[8] Lord, F. M. (1980). Applications of item response theory to practical testing problems. Hillsdale, NJ: Lawrence Erlbaum Associates.

[9] Vale, C. D. (1986). Linking item parameters onto a common scale. Applied Psychological Measurement, 10, 333-344. 